\title{
Two Dimensional Ultrasound and Doppler in Assessment of Adnexal Masses in Correlation to Histo Pathological Analysis
}

\author{
Polysetty Obuleswar Prasad ${ }^{1}$, O. Sreedhar Babu' ${ }^{2}$, G.V.Prasad ${ }^{3}$, D.R. Anand ${ }^{4}$ \\ ${ }^{1}$ Assistant Professor, ${ }^{2}$ Assistant Professor, ${ }^{3}$ Associate Professor, ${ }^{4}$ Professor \& HOD Department of Radiodiagnosis, Sri \\ Venkateswara Medical College Tirupathi, India \\ Corresponding author: Dr.Polysetty Obuleswar Prasad,MD, Assistant Professor, Department of Radiodiagnosis, Sri \\ Venkateswara Medical College, Tirupathi, India
}

DOI: http://dx.doi.org/10.21276/ijcmsr.2020.5.1.25

(c) BY-NC-ND

How to cite this article: Polysetty Obuleswar Prasad, O. Sreedhar Babu, G.V.Prasad, D.R. Anand. Two dimensional ultrasound and doppler in assessment of adnexal masses in correlation to histo pathological analysis. International Journal of Contemporary Medicine Surgery and Radiology. 2020;5(1):A107-A114.

\section{A B S T R A C T}

Introduction: Doppler US is useful in cases with an apparent solid area or septum, while transabdominal US is helpful for larger masses or those located superiorly or laterally in the pelvis, transvaginal US provides optimal visualization of most adnexal diseases. The aim of this study was to evaluate the diagnostic value of ultrasonography in adnexal masses and its correlation to histopathological diagnosis.

Material and methods: The present study is a two years study from October 2017 to October 2019 carried out on 50 patients with suspected adnexal masses from the department of Gynecology. The cases were recruited from Sri Venkateswara medical college, Tirupathi. All patients underwent ultrasound and the final diagnosis was made by histopathological examination in 50 cases. All histopathology reports were reviewed. The findings of sonography was correlated to histopathological findings, which were taken as gold standard.

Results: In the present study out of 50 cases, 37 cases (74\%) were diagnosed as benign in US, 13 cases (26\%) were malignant on correlation to histopathological analysis. 40 cases (80\%) were benign, 10 cases (20\%) were malignant. 3 cases (6\%) were assumed as malignant in US, but actually not in histopathology. The overall sensitivity was $92.5 \%$ and specificity was $100 \%$, positive predictive value was $100 \%$ and negative predective value $76.92 \%$.

Conclusion: 2D US and Doppler study with a good equipment when appropriately performed by an experienced radiologist, using a proper methodology and standard guidelines has proved to be a very useful highly diagnostic and a reliable method with good sensitivity and specificity.

Keywords: Two Dimensional Ultrasound, Doppler, Adnexal Masses, Histo Pathological Analysis

\section{INTRODUCTION}

Adnexal masses are considered a group of the most common diseases in gynecology. Ovarian tumors alone, represent two thirds of these cases. Ovarian neoplasms present an increasing challenge to the physician, and ovarian cancer being the most lethal of all gynecological cancers, presents late and responds poorly to treatment. Malignant ovarian tumors are the fourth most common cause of death in women. Approximately $4-24 \%$ of adnexal masses in premenopausal women and $39-63 \%$ in postmenopausal women are malignant. ${ }^{1}$ Ultrasonography (US) remains the imaging modality most frequently used to detect and characterize adnexal masses. Although evaluation is often aimed at distinguishing benign from malignant masses, the majority of adnexal masses are benign. About $90 \%$ of adnexal masses can be adequately characterized with US alone.

Adequate characterization of an adnexal mass is important both to determine which patients need surgery and to help define the type of surgery and whether a surgical subspecialist is needed. In general, US features that indicate malignancy include as Solid component (particularly if there is visible flow in it at Doppler evaluation), Thick septa. Ascites, Doppler criteria that indicate malignancy as Increased vascularity. US demonstration of a solid component within a cystic mass is the most important predictor of malignancy, and conversely, malignancy is very unlikely in the absence of a solid component. Terminology to describe the solid component varies and also includes papillary projection, excrescence, vegetation, and nodule. It has been suggested that small solid areas that protrude $3 \mathrm{~mm}$ or more from the cyst wall be considered as papillary projection all irregularities due to a collapsing cyst can simulate small solid nodules that may be misconstrued for malignancy. The completely solid adnexal mass is another potential problem. Most commonly, such a mass is due to a pedunculated uterine leiomyoma or an ovarian fibroma. ${ }^{2}$

The majority of epithelial ovarian malignancies has a cystic component and is rarely completely solid. There are sporadic exceptions, but the majority of completely (ie, 100\%) solid, solitary adnexal masses are benign in our experience, and other authors have a similar opinion. Ovarian malignancies 
that are most likely to manifest as solid or nearly completely solid masses include metastases, lymphoma, neoplasms of the sex cord- stromal group, and other rare malignancies such as malignant teratomas or dysgerminomas. Septa in a cystic ovarian mass are evidence of a neoplasm and are more likely to indicate malignancy if they are greater than $2-3 \mathrm{~mm}$ in thickness or have detectable flow on Doppler US scans. A cystic ovarian mass with septa but without a solid component is likely to be a benign neoplasm, though occasionally may be malignant when there are a very large number of septa. A cystic mass with multiple, smooth, thin septa and no nodularity is suggestive of a mucinous cystadenoma. ${ }^{3}$

Ascites, an indirect indicator of malignancy, occurs with peritoneal tumor spread. Ascites may allow peritoneal implants to be seen. Although a small amount of fluid in the cul-de sac is normal in premenopausal women, an increased risk of malignancy has been reported if it measures more than $15 \mathrm{~mm}$ in anteroposterior dimension.

Doppler ultrasound is a technique used to determine vascular indices from computer algorithms. These vascular indices provide an indication for the numbers of vessels that can be detected within the organ and the number of blood cells that are transported per minute. There is a general trend toward lower pulsatility index, lower resistive index, and higher velocity in malignant neoplasms as opposed to benign neoplasms. However, because of the substantial overlap of these spectral doppler parameters in benign and malignant lesions, they have little to no role in the characterization of adnexal masses. ${ }^{4}$ Combined morphological and vascular imaging obtained by pelvic ultrasonography and power doppler appears to further improve preoperative assessment of adnexal masses.

Current study aimed to study the Ultrasonographic and Doppler findings in various adnexal masses and to know the sensitivity and specificity of Ultrasonography and Doppler Findings in evaluation of adnexal masses and to correlate the diagnostic accuracy of Ultrasound with Pathological diagnosis.

\section{MATERIAL AND METHODS}

This was a correlative study, done on 50 patients with signs and symptoms of adnexal masses referred to the Radiology department. The study is done for a period of 2 years from October 2017 to October 2019.

The cases with signs and symptoms of adnexal masses were referred for Colour Doppler Ultrasonography study to the department of Radio Diagnosis in Sri venkateswara Medical College, Tirupathi. It is confirmed by the Histopathology study by sending the sample of the mass to the pathology department in our college. Histopathology examination was done by using Hematoxylin\& Eosin stain in the department of Pathology, Sri Venkateswara medical college.

Patients were referred from the Gynecology Department with following inclusion and exclusion criteria for this study.

\section{Inclusion criteria}

Female patients [pre pubertal to post menopausal] of all age groups presenting with adnexal mass.

Patients presenting with Ovarian mass.

Patients with mass in Fallopian tubes.

\section{exclusion criteria}

Patients with infective etiology like PID etc.

Patients with Ectopic pregnancy.

Patients with Masses from GIT Pathology.

All of them were subjected to Ultrasonography with $3.5 \mathrm{MHz}$ probe. Ultrasound was performed with the use of ESOATE My Lab Class C Diagnostic Ultrasound System. Observations included size, shape and echo texture of the adnexal masses in sagittal and transverse planes. IOTA scoring system was applied to differentiate benign and malignant ovarian tumors.

All eligible patients were properly counselled and gave informed consent before entry into the study. Collected data was analyzed by comparing it with histopathology to know sensitivity, specificity, positive predictive value, negative predictive value and diagnostic accuracy of the imaging modalities using Statistical Package for the Social Sciences (SPSS) version 24.

\section{RESULTS}

Ultrasound scan was performed in 50 patients who presented

\begin{tabular}{|l|c|c|}
\hline Age Group (years) & No. of cases & \% of Cases \\
\hline $11-20$ & 5 & 10 \\
\hline $21-30$ & 7 & 14 \\
\hline $31-40$ & 15 & 30 \\
\hline $41-50$ & 12 & 24 \\
\hline $51-60$ & 8 & 16 \\
\hline $61-70$ & 3 & 6 \\
\hline Total & 50 & 100 \\
\hline \multicolumn{2}{|c|}{ Table-1: Age wise incidence of adnexal masses } \\
\hline
\end{tabular}

\begin{tabular}{|l|c|}
\hline Clinical Diagnosis & No. of Cases \\
\hline Serous Cystadenocarcinoma & 7 \\
\hline Mucinous Cystadenocarcinoma & 5 \\
\hline Dysgerminoma & 1 \\
\hline Total & 13 \\
\hline Table-2: Number of malignant tumors diagnosed in Ultrasound \\
\hline
\end{tabular}

\begin{tabular}{|l|c|c|}
\hline Adnexal masses & $\begin{array}{c}\text { No. of } \\
\text { Cases }\end{array}$ & \% of Cases \\
\hline Benign masses & 37 & $74 \%$ \\
\hline Malignant masses & 13 & $26 \%$ \\
\hline Total & 50 & $100 \%$ \\
\hline Clinical Diagnosis & 18 & 36 \\
\hline Benign serous cystadenoma & 18 & 18 \\
\hline Benign Mucinous Cystadenoma & 9 & 14 \\
\hline Mature Cystic Teratoma & 7 & 10 \\
\hline Serous Cystadenocarcinoma & 5 & 8 \\
\hline Mucinous Cystadenocarcinoma & 4 & 6 \\
\hline Hemorrhagic cyst & 3 & 4 \\
\hline Endometrioma & 2 & 2 \\
\hline Ovarian Fibroma & 1 & 2 \\
\hline Dysgerminoma & 1 & 100 \\
\hline Total & 50 & \\
\hline $\begin{array}{l}\text { Table-3: Distribution of adnexal masses diagnosed in Histopa- } \\
\text { thology }\end{array}$ \\
\hline
\end{tabular}


with symptoms, and signs of adnexal mass. The results are enumerated in table- 1 . The table- 1 shows the maximum number of cases were in the age group of 31- 50 years and the minimum number were in the age group of $61-70$ years. In this present study out of 50 cases, 37 cases were diagnosed as benign masses in ultrasound and 13 cases were malignant (figure-1).

In this present study out of 50 cases, 40 cases were diagnosed as benign masses in histopathology and 10 cases were malignant (table-3,4).

\section{Statistics}

Value

Sensitivity

92.50\%

Specificity

$100.00 \%$

Positive Predictive Value $\quad 100.00 \%$

Negative Predictive Value $\quad 76.92 \%$

In the present study total number of cases were 50 . On ultrasound 37 cases were diagnosed as benign and 13 were

\begin{tabular}{|l|c|c|}
\hline Ovarian Lesions & No. of Cases & \% of Cases \\
\hline Benign positive & 40 & $80 \%$ \\
\hline Malignancy positive & 10 & $20 \%$ \\
\hline Total & 50 & $100 \%$ \\
\hline Table-4: Total number of Adnexal masses diagnosed in histo- \\
pathology \\
\hline
\end{tabular}

\begin{tabular}{|l|l|c|c|c|}
\hline \multicolumn{2}{|l|}{ Adnexal masses } & \multicolumn{2}{|c|}{ HPE } & \multirow{2}{*}{ Total } \\
\cline { 3 - 4 } \multicolumn{2}{|c|}{} & Benign & Malignant & \\
\hline \multirow{3}{*}{ Ultrasound } & Benign & 37 & 0 & 37 \\
\cline { 2 - 5 } & Malignant & 3 & 10 & 13 \\
\hline Total & 40 & 10 & 50 \\
\hline
\end{tabular}

Table-5: Correlation of ultrasound diagnosis of Adnexal masses to Histopathological diagnosis

\begin{tabular}{|l|c|c|c|}
\hline Age in years & $\begin{array}{c}\text { No of benign } \\
\text { cases }\end{array}$ & $\begin{array}{c}\text { No of malig- } \\
\text { nant cases }\end{array}$ & $\begin{array}{c}\text { Total no. of } \\
\text { cases }\end{array}$ \\
\hline $11-20$ & 5 & 0 & 5 \\
\hline $21-30$ & 7 & 1 & 8 \\
\hline $31-40$ & 15 & 0 & 15 \\
\hline $41-50$ & 11 & 0 & 11 \\
\hline $51-60$ & 2 & 6 & 8 \\
\hline $61-70$ & 0 & 3 & 3 \\
\hline Total & 40 & 10 & 50 \\
\hline Table-6: Incidence of benign and malignant cases according to \\
\multicolumn{4}{|c}{ various age groups. } \\
\hline
\end{tabular}

\begin{tabular}{|l|c|c|c|c|c|c|}
\hline & $\mathbf{1 1 - 2 0}$ & $\mathbf{2 1 - 3 0}$ & $\mathbf{3 1 - 4 0}$ & $\mathbf{4 1 - 5 0}$ & $\mathbf{5 1 - 6 0}$ & $\mathbf{6 1 - 7 0}$ \\
\hline Serous cystadenoma & - & 2 & 7 & 7 & 2 & - \\
\hline Mucinous cystadenoma & - & - & 5 & 4 & - & - \\
\hline Cystic teratoma & 3 & 4 & - & - & - & - \\
\hline Serous cystadeno carcinoma & - & - & - & - & 3 & 2 \\
\hline Mucinous cystadeno carcinoma & - & - & - & 0 & 3 & 1 \\
\hline Hemorrhagic cyst & - & - & 3 & - & - & - \\
\hline Endometrioma & 2 & - & - & - & - & - \\
\hline Ovarian fibroma & - & 1 & - & - & - & - \\
\hline Dysgerminoma & - & 1 & - & - & - & - \\
\hline \multicolumn{2}{|l|}{ Table-7: Distribution of adnexal mass according to the age } & \\
\hline
\end{tabular}

CASE No.1: DERMOID CYST

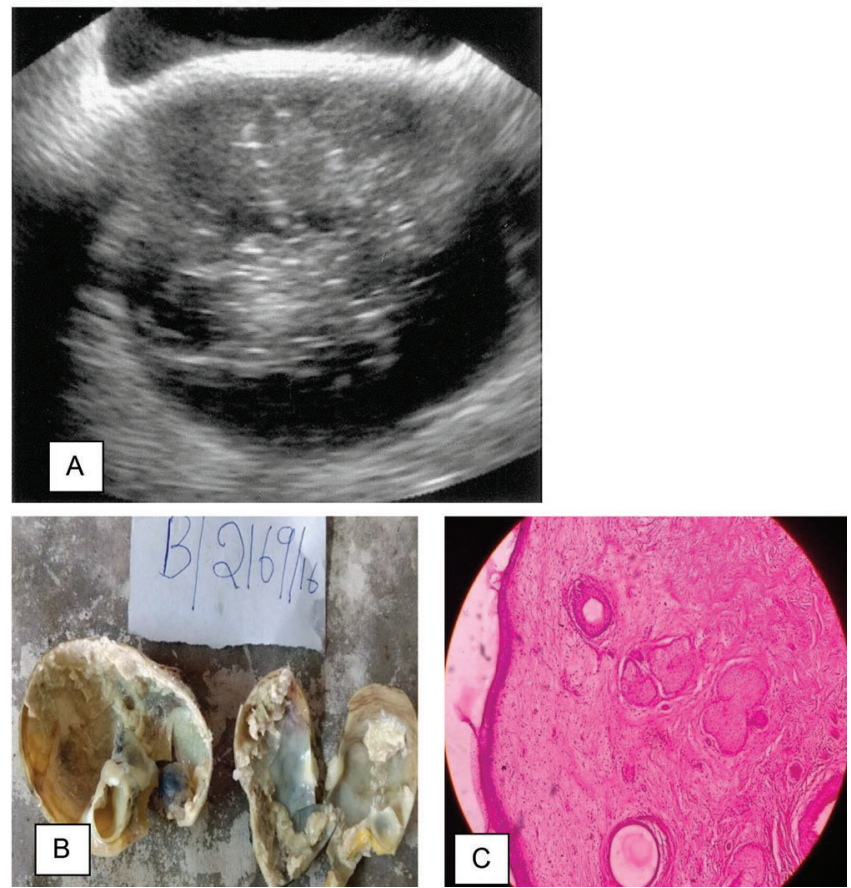

Figure-1: A case of dermoid cyst

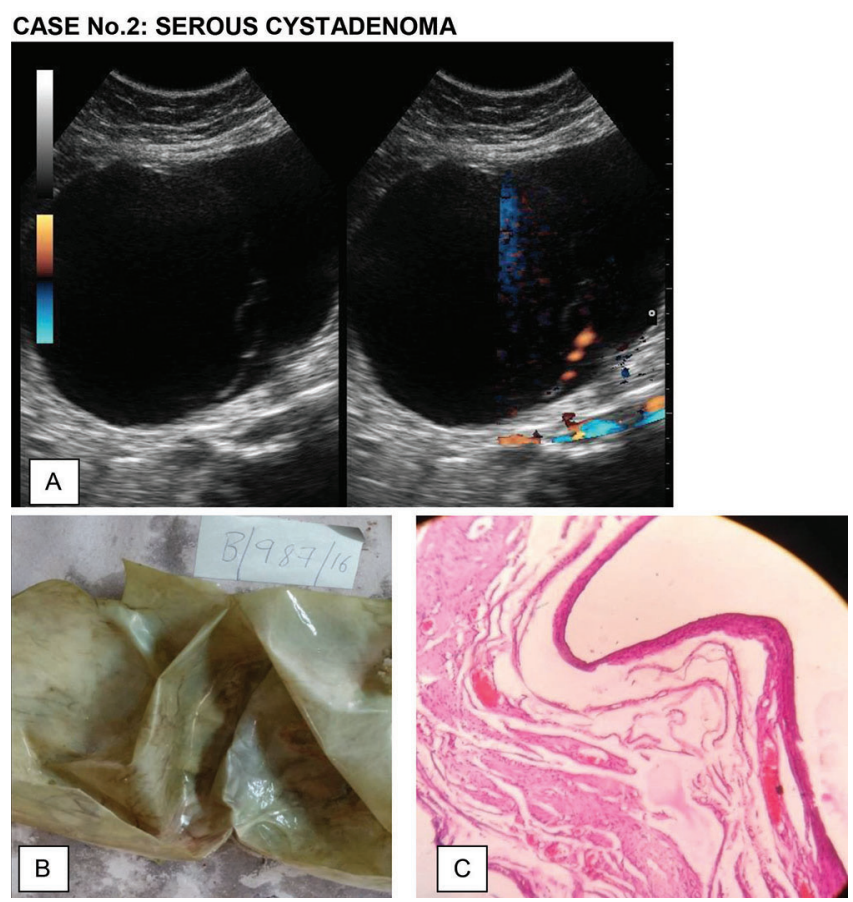

Figure-2: A case of serous cystadenoma 


\section{CASE No.3: SEROUS CYSTADENOCARCINOMA}

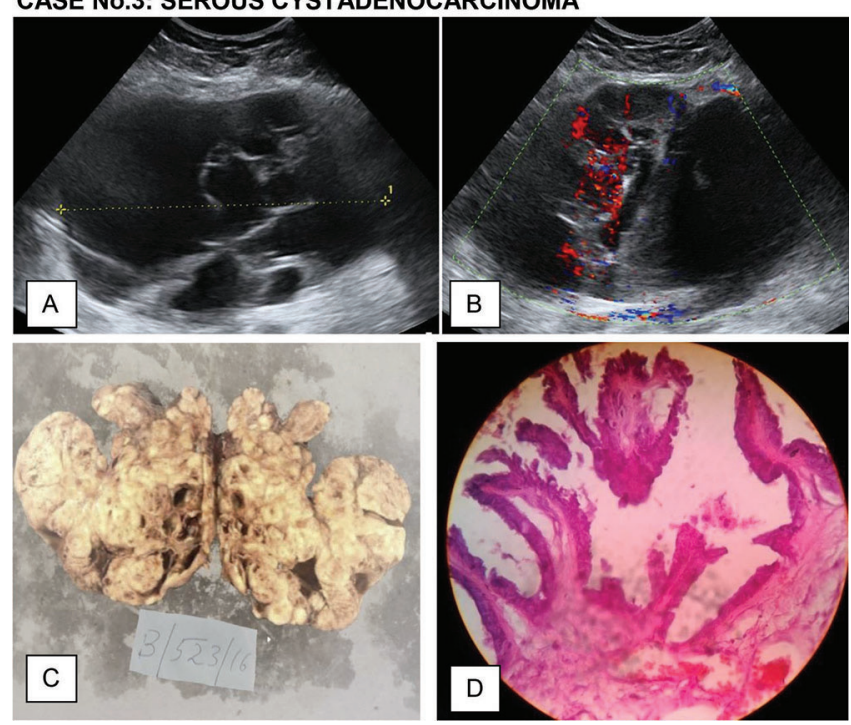

Figure-3: A case of serous cystadenocarcinoma
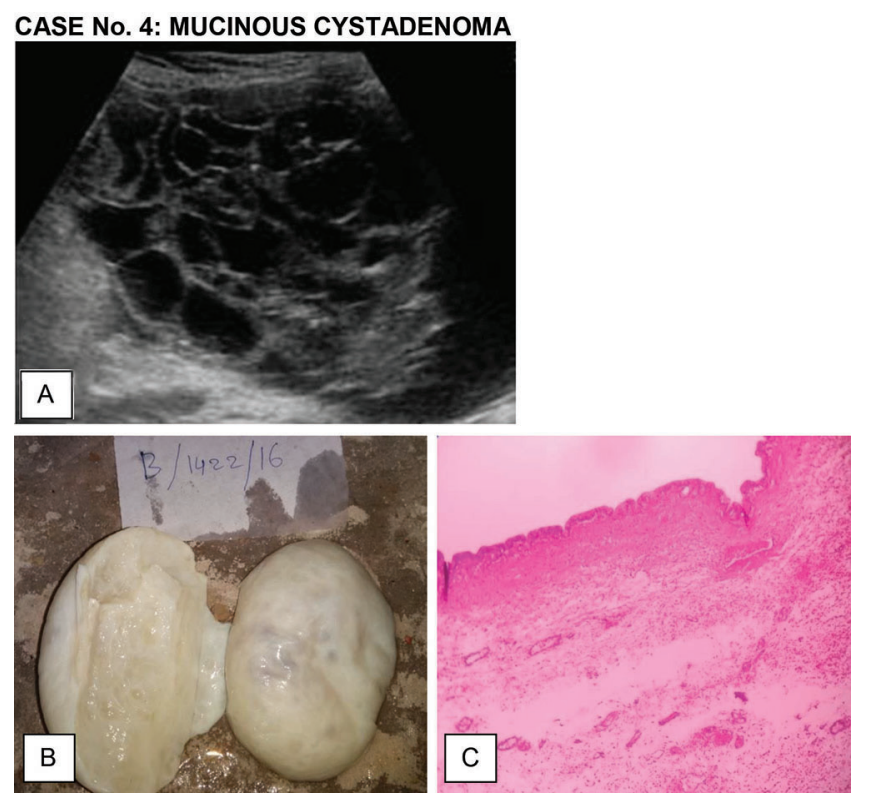

Figure-4: A case of mucinous cystadenoma

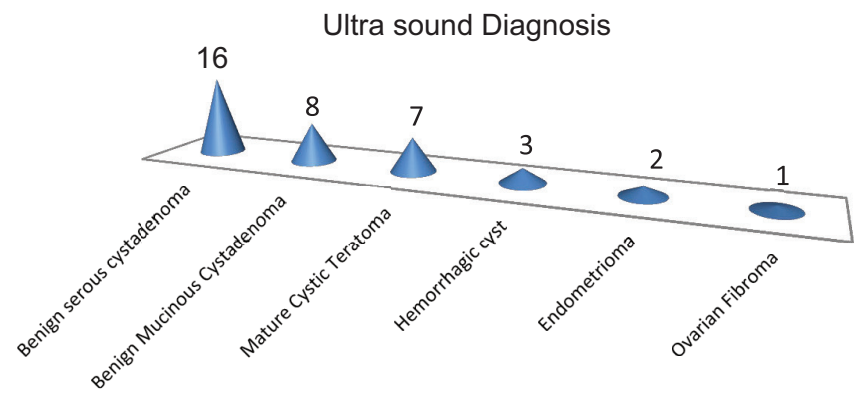

Graph-1: Number of benign tumours diagnosed in ultrasound

malignant. On histopathology 40 cases were confirmed as benign and only 10 cases were malignant (table-5).

In this present study, Ultrasonography showed an overall sensitivity of $92.50 \%$ and specificity of $100 \%$. Positive predictive value $100 \%$ and negative predictive value $76.92 \%$ in comparison to the histopathological findings.
Case-1: Dermoid Cyst

Transabdominal US image shows a heterogeneous mass containing echogenic reflectors representing hair. There is no evidence of calcification or fat.

Grossly, the ovarian mass appears bulky and have smooth external surface. On c/s the mass tends to be solid with Rockitonsky protuberance and shows cartillage, hair, sebacious material along with areas of necrosis and hemorrhage.

Histopathology shows squamous epithelial lining, cartilage, hair shaft surrounded by sebaceous glands

\section{Case-2: Serous Cystadenoma}

Transabdominal US show hypoechoic unilocular smooth walled cystic lesion with thin septations. Minimal flow on power doppler.

Groosly, ovary shows serous filled cyst, cut section shows thin fibrous wall covering cyst.

Histopathology shows fibrous cyst wall lined by flattened epithelium.

\section{Case No.3: Serous Cystadenocarcinoma}

Transabdominal US show Ill defined hypo echoic cystic lesion with papillary projections, thick septations and solid components.

On color doppler the lesion shows internal vascularity in the solid components and in thick septations.

Grossly ovarian mass appears as large solid and cystic mass, irregular and nodular surface. On cut section shows large cystic area with large solid/papillary growth.

Histopathological examination shows Multilayered epithelium with papillary areas. Stratification of epithelium, nuclear atypia, increased complexity of stromal papillae. Focal invasion of stroma seen.

\section{Case No. 4: Mucinous Cystadenoma}

Transabdominal US show Ill defined multilocular cystic lesion with numerous thin septations with low-level internal echogenicity due to increased mucin content

Grossly, ovary appears as greywhite glistering mass. Cut section shows cyst filled with grey white mucin material.

Histopathology shows fibrous cyst wall lined by columnar epithelium filled with mucinous material.

\section{DISCUSSION}

Ovarian cancer is the second most common female reproductive cancer, preceded only by the cancer of the uterine corpus. More women die from ovarian cancers, as it corresponds to the highest mortality rate in developed countries. As a result, many patients undergo major surgery because of the fear of missing an ovarian cancer. On the contrary, many women with advanced ovarian cancer undergo insufficient primary surgeries at local hospitals, and the suboptimal intervention affects prognosis and increases patient morbidity.

Malignant ovarian tumors are diagnosed at an advanced stage in $75 \%$ of cases and are associated with the highest mortality figures of all gynecological cancers. ${ }^{5}$ In evaluation of adnexal masses USG is the primary modality for diagnosing pelvic masses. ultrasonography is definitely an important noninvasive investigation and is helpful in diagnosing most cases 
of adnexal masses, the histopathological examination of specimen obtained from laparotomy/laparoscopy of adnexal mass is the gold standard for confirming the diagnosis. ${ }^{6}$ In present study supports the hypothesis that ultrasonographic evaluation and Doppler U/S might help to improve preoperative differentiation between benign and malignant ovarian tumors.

\section{Age Incidence}

In the present study, the mean age of studied cases was 38.76 , the maximum age was 63 and the minimum was 16 . In the present study the incidence of various adnexal masses (50 in number) according to age group

is 5 benign cases in 11-20 years of age, 7 benign and 1 malignant cases in 21-30 years of age, 15 benign cases in 31-40 years of age, 11 benign cases in 41-50 years of age, 2 benign and 6 malignant cases in 51-60 years of age, 3 malignant cases in 61-70 years of age are seen (table-6).

The consistency of the masses was 58\% cystic, $38 \%$ solid with cystic component and $4 \%$ solid. These masses were $56 \%$ unilocular and $42 \%$ multilocular. The inner wall of the masses was regular in $66 \%$ of masses, nodular in $32 \%$. Thin septa were found in $32 \%$ of masses, $28 \%$ of them were found to be thick septations.

Ascites was found in 12 (24\%) cases, in three of these cases was massive (reaching hepatorenal pouch). Application of doppler waves on these adnexal masses revealed that 14 of masses were vascular.

Ultrasonographic subjective impression (regarding malignancy) was detected for each mass. Depending on the $2 \mathrm{D}$ gray-scale U/S characteristics and vascular pattern by doppler waves, masses revealed that 40 of them were benign. Histopathological analysis of the surgically excised 10 masses were malignant. The most common benign tumor in this study is Benign serous Cystadenoma (18 cases). The next most common masses are Mucinous Cystadenoma, Mature cystic teratoma, follicular cysts. In this study, by correlation of all previous tools and findings with the histopathological analysis found that clinical evaluation including various clinical parameters had a low sensitivity as regard the differentiation between benign and malignant ovarian tumors, a finding that was previously concluded by Roman et $\mathrm{al}^{7}$ Tenderness was encountered in some uncomplicated benign masses, in all complicated benign masses and also in some malignant tumors. This finding agreed with who found that benign tumors became tender probably due to the tense nature of their contents. On the other hand, infiltration of the capsule by malignant cells may account for tenderness in malignant tumors. The incidence of malignancy in this study was found to be $26 \%$.

According to various studies, most ovarian tumors (80\% to $85 \%)$ are benign and two-thirds of these occur in women in reproductive age. The chance that an ovarian tumor is malignant in a patient younger than 40 years of age is about $7 \%$. Approximately $4-24 \%$ of adnexal masses in premenopausal women and $39-63 \%$ in postmenopausal women are malignant. ${ }^{8}$ In this study the incidence of malignancy is $2 \%$ in reproductive age group.

Most of benign masses were found to be cystic (72.0\%).
9 of malignant masses were found to be cystic with solid component (18\%) and one case is solid (2\%).

Kupesic ${ }^{9}$ generally used ultrasound for discriminating the benign from malignant lesions, also to determine the histological type of tumors. Criteria to distinguish includes the locularity and size of the cyst, the thickness of the cyst wall and any septations present, the presence of solid nodules or papillary projections, blood flow in any solid component of the cyst especially with low resistance and the presence of ascites.

No one characteristic confirms malignancy but rather it is a subjective decision taking into account characteristics on ultrasound as well as the patient's age and other risk factors. Malignancy is more likely when the cyst is $>10 \mathrm{~cm}$, septations $>2-3 \mathrm{~mm}$ thick, presence of solid components with blood flow, the blood flow on Doppler with a resistive index of $<0.4$, or a pulsatility index $<1.0$ and ascites in the postmenopausal patient.

If all the other ultrasound parameters are reassuring; however, a unilocular lesion without internal echo or papillary excrescences is highly unlikely to be malignant regardless of the size or age of patient. Thick septations were found in $26 \%$ of malignant cases and only in $6 \%$ of benign cases. Inner wall was smooth in 33 of benign masses while it was nodular in all malignant masses. The mean largest dimension in $\mathrm{cm}$ by USG for benign masses (40 cases) was 7.2 and for malignant masses (10 cases) was 12 . 3. Ascites was found in $76.9 \%$ of malignant cases and $5 \%$ of benign masses.

Vascular indices were calculated for each mass. RI and PI, revealing a high diagnostic value in predicting malignancy in various adnexal masses.

In present study the ultrasound characteristic features of various Adnexal masses were studied. 28 benign masses were unilocular, No malignant mass was unilocular. 8 benign masses were multilocular, 13 malignant masses were multilocular.

Solid component was seen in 8 benign and 13 malignant masses. Thin septations were seen in 14 benign and 2 malignant masses. Thick septations were seen in 3 benign and 13 malignant masses. Smooth inner wall is seen in 33 benign and 1 malignant mass.

Nodular inner wall was seen in 3 benign and 13 malignant masses. Ascites was seen in 2 benign and 10 malignant masses. Internal vascularity was seen in 2 benign and 12 malignant masses.

In the present study the diagnosis of adnexal masses based on ultrasound characteristics was correlated to histopathological diagnosis. 16 out of 18 cases of Serous cystadenoma were diagnosed in ultrasound. Remaing two cases were wrongly diagnosed as malignant cases in ultrasound. 8 out of 9 cases of Mucinous cystadenoma were diagnosed in ultrasound. Remaining one case was wrongly diagnosed as malignant mass in ultrasound. 7 cases of serous cystadenocarcinoma were diagnosed in ultrasound, two of them were benign as confirmed by histopathological diagnosis. 5 cases of Mucinous cystadenocarcinoma were diagnosed in ultrasound, one of them was benign as confirmed by histopathological diagnosis. 


\section{Benign serous cystadenoma}

In the present study Benign serous cystadenomas have accounted for 18 cases (36\%). Among 18 cases, 2 cases in 21 30 years of age, 7 cases in 31-40 years of age, 7 cases in $41-50$ years of age, 2 cases in 51-60 years of age.

In the present study 16 cases of Benign serous cystadenoma were showed unilocular hypo echoic lesions with smooth inner wall structure without any septations. These were confirmed as benign in Histopatology diagnosis. 2 cases were showed multi locularity, thick septations with internal vascularity leading to suspicion of malignancy. These two masses were confirmed as Benign serous cystadenoma in Histopathology diagnosis.

\section{Benign Mucinous Cystadenoma}

In the present study Benign Mucinous cystadenoma have acconted for 9 cases (18\%). Among 9 cases, 5 cases in 31-40 years of age, 4 cases in 41-50 years of age.

In the present study 8 cases were showed multilocular hypo echoic lesions with internal echoes with smooth inner wall structure with thin septations. These were confirmed as benign in Histopatology diagnosis. 1 case showed multi locularity, thick septations with internal vascularity leading to suspicion of malignancy. This mass was confirmed as Benign Mucinous Cystadenoma in Histopatology diagnosis.

\section{Mature Cystic Teratoma}

In the present study Mature cystic teratoma have accounted for 7 cases (14\%) Among 7 cases, 3 cases in 11-20 years of age, 3 cases in 11-20 years of age. In the present study 7 cases were showed unilocular mixed echoic smooth walled cystic lesion with solid content and posterior acoustic shadowing. Few of them showed fluid fluid levels indicating result of layering of serous fluid and sebum.

\section{Serous Cystadenocarcinoma}

In the present study Serous Cystadenoma have accounted for 5 cases (10\%). Among 5 cases, 3 cases in 51-60 years of age, 2 case in 61-70 years of age.

In the present study 5 cases were showed multi locularity, thick septations with internal vascularity with ascites leading to suspicion of malignancy, diagnosed as Serous Cystadenocarcinoma. These were confirmed on histopathology diagnosis.

\section{Mucinous Cystadenocarcinoma}

In present study Mucinous Cystadenocarcinoma have accounted for 4 cases (8\%). Among 4 cases 3 cases in 5160 years of age, 1 case in 61-70 years of age. In the present study 4 cases were showed multi locularity, thick septations with internal vascularity with ascites leading to suspicion of malignancy, diagnosed as Mucinous Cystadenocarcinoma. These were confirmed on histopathology diagnosis.

\section{Hemorrhagic cyst}

In present study Hemorrhagic cyst have accounted for 3 cases (6\%). All cases are seen in 31-40 years of age. In the present study cases were showed unilocular ill defined cystic lesion with irregular wall. lace like reticular echogenic appearance showing typical of hemorrhagic cyst.

\section{Endometrioma}

In present study Endometrioma have accounted for 2 cases (4\%). All cases are seen in 11-20 years of age. In present study cases were showed unilocular smooth walled cystic lesion with ground glass echogenicity without any solid contents.

\section{Ovarian Fibroma}

In present study Ovarian Fibroma have accounted one case (2\%). One case is seen in 21-30 years of age. In the present study case showed ill defined solid hypoechoic lesion with minimal color flow noted on dopper.

\section{Dysgerminoma}

In present study Dysgerminoma have accounted one case (2\%). one case is seen in 21-30 years of age In the present study case showed multilocular mixed echogenic irregular cystic lesion noted with nodular wall and thick septations. On color Doppler septations shows significant vascularity. This study supports the hypothesis that ultrasonographic evaluation of tumor angiogenesis might help to improve differentiation between benign and malignant ovarian tumors detected in screening trials, as stated by Carmeliet et al. ${ }^{10}$

In the present study, pulsed wave doppler and color doppler applications correctly diagnosed false positive cases of clinical evaluation, US lonely. This was by detecting peripheral flow with low doppler indices and high vascular indices. So, combination of various diagnostic modalities with doppler wave application increases their specificity and diagnostic accuracy.

This proves that the doppler wave application should be used as a complementary tool in the diagnosis of ovarian tumors. Folkman et $\mathrm{al}^{11}$ described the importance of angiogenesis for tumor growth. In general, both indices tended to be lower in malignant masses than in benign masses. ${ }^{12}$ There is no cutoff value with both high sensitivity and high specificity for malignancy, precluding the use of any single cutoff value as a sole designator of the malignant or benign nature of an ovarian mass.

Guerriero et $\mathrm{ll}^{11}$ concluded that at least one of the two doppler techniques, pulsed wave or color doppler, should be used in conjunction with gray-scale imaging in order to decrease the false positive rate of gray-scale put the increasing evidence that both indices demonstrate considerable overlap between malignant and benign ovarian masses and so they limited the usefulness of pulsed doppler ultrasound in differentiating these lesions.

The results of a study carried out by Fleischer et $\mathrm{al}^{12}$ showed a statistically significant difference between vascularity in benign lesions, which tended to be peripheral and that in malignant lesions which tended to be central.

Cohen et $\mathrm{al}^{13}$ published a study on 71 women with a known complex pelvic mass who were referred for a preoperative ultrasound evaluation with both TVS and power doppler. They correctly identified all 14 ovarian malignancies (2 FIGO stage I, 2 stage II, 7 stage III, and 3 metastatic colon) by both TVS and power doppler imaging having sensitivity of $100 \%$.

This seems to be an important finding, because Bell et al. had established that an increase in cancer detection at stage I 
from $25 \%$ to $50-75 \%$ might result in about $20-40 \%$ reduction in ovarian cancer mortality at five years. It was mentioned in literature that pattern recognition by an experienced sonologist is an excellent method for discriminating between benign and malignant adnexal masses and should probably be regarded as the standard method for preoperative classification of adnexal masses.

However, the ability to discriminate between benign and malignant adnexal masses using vascular pattern recognition increases with increasing experience, and in daily clinical practice, it is impossible to ask an expert's opinion on every adnexal mass.

Valentin ${ }^{14}$ recommended to refer cases with adnexal tumors to distinguish between benign and malignant adnexal tumors, with an expected accuracy of $95 \%$. But also he conducted a study on the use of pattern recognition for discrimination between benign and malignant adnexal masses by non-expert ultrasound operators, where results reached a sensitivity and specificity with regard to malignancy of $86 \%$ and $80 \%$, respectively.

Yazbek et al stated the importance of the quality of ultrasonography machine and its resolution, in addition to the experienced operator, in the management of patients with suspected ovarian cancer in a tertiary gynecologic center and how it results in a significant decrease in the number of major staging procedures and a shorter patient hospital stay. ${ }^{15}$ An accurate diagnosis is essential to provide optimal treatment, as the rupture of a Stage I ovarian cancer during surgery may worsen the prognosis.

Because of the low incidence of ovarian cancer in clinical practice, reported to be approximately one case per 2,500 women per year, it has been estimated that a screening test with $100 \%$ sensitivity and $99.6 \%$ specificity is needed to achieve a positive predictive value of $100 \%$, i.e. to limit the number of unnecessary surgical procedures to nil for each detected case of ovarian cancer. ${ }^{16}$

In the present study, Ultrasonography showed sensitivity of $98.43 \%$ and specificity of $87.84 \%$ in benign cases, where as sensitivity of $89.07 \%$ and specificity of $98.38 \%$ in malignant cases. Ultrasound is the main diagnostic imaging modality prior to treatment. Improved detection and characterization of adnexal mass contributes to better diagnostic accuracy and consequently reduction of false-positive findings and invasive procedures, which leads to a significant reduction of morbidity and mortality.

\section{CONCLUSION}

In the present study it was concluded that 2D US with doppler study had increased its specificity to $100 \%$ in the prediction of ovarian malignancy With the use of different modalities of ultrasound and doppler wave technology can precisely help in predicting malignancy in various adnexal masses.

The present study had concluded that 2D US and Doppler study with a good equipment when appropriately performed by an experienced radiologist, using a proper methodology and standard guidelines has proved to be a very useful highly diagnostic and a reliable method with good sensitivity and specificity.

\section{REFERENCES}

1. Myers, E.R., L.A. Bastian, L.J. Havrilesky, S.L. Kulasingam, M.S. Terplan, K.E. Cline, R.N. Gray and D.C. McCrory. Management of adnexal mass. Evid. Rep. Technol. Assess. 2006;130(1):1-14.5.

2. Oh SN, Rha SE, Byun JY, et al. MRI features of ovarian fi bromas: emphasis on their relationship to the ovary. Clin Radiol 2008;63(3):529 - 535 .

3. Valentin, L. Use of morphology to characterize and manage common adnexal masses. Best Pract. Res. Clin. Obstet. Gynaecol. 2004;18(5):71-89.

4. Buy, J.N., M.A. Ghossain, D. Hugol, K. Hassen and C. Sciot. Characterization of adnexal masses: combination of color Doppler and conventional sonography compared with spectral Doppler analysis alone and conventional sonography alone. AJR 1996;166(2):385-393.

5. Jemal, A., R. Siegel, E. Ward, T. Murray, J. Xu and M.J. Thun. Cancer statistics. CA Cancer J. Clin. 2007;57(4):43-66.

6. Bhagde AD et al. An analytical study of 50 women presenting with an adnexal mass Int J Reprod Contracept Obstet Gynecol. 2017;6(1):262-265.

7. Roman, L.D., I.M. Laila, S.M. Stein, L.S. Barin, G. Susan and P.C. Mattow. Pelvic examination, tumor marker level, gray scale and Dopplersonography in the prediction of pelvic cancer. Obstet. Gynecol., 1997;89(4): 493-500.

8. Vasilev, S.A., J.B. Schlaertr, J. Campeau and C.P. Morow. Serum CA125 levels inpreoperative evaluation of pelvic masses. Obstet. Gynecol. 1988;72(1): 659-64.

9. Kurjak, A., S. Kupesic and V. Simunic. Ultrasonic assessment of the peri- and postmenopausal ovary. Maturitas. 2002;41(5):245-54.

10. Carmeliet, P. and R.K. Jain. Angiogenesis in cancer and other diseases. Nature 2000;407(6):249-57.

11. Guerriero, S., J.L., Alcazar, S. Ajossa, et al. Comparison of conventional color Doppler imaging and power Doppler imaging for the diagnosis of ovarian cancer: results of a European study. Gynecol. Oncol. 2001;83(2): 299-304.

12. Fleischer, A.C., J.A. Cullinan, D.M. Kepple and L.L. Williams. Conventional and color Doppler transvaginal sonography of pelvic masses: A comparison of relative histologic specificities. J. Ultrasound Med. 1993;12(4):705-12.

13. Cohen, L., et al. Is transvaginal ultrasound effective for screening asymptomatic women for the detection of early-stage epithelial ovarian carcinoma? Gynecol. Oncology, 2001;77(1):347-349.

14. Valentin,L.,D.Jurkovic,B.Van Calster,A.TestaC.Van Holsbeke, T. Bourne, I. Vergote, S. Van Huffel and D. Timmerman. Adding a single CA-125 measurement to ultrasound performed by an experienced examiner does not improve discrimination between benign and malignant adnexal masses. A prospective international multicentre study of 809 patients. 
Ultrasound Obstet. Gynecol. 2009;34(2): 345-354.

15. Yazbek, J., S.K. Raju, J. Ben-Nagi, et al. Effect of quality of gynaecological ultrasonography on management of patients with suspected ovarian cancer: a randomized controlled trial. Lancet. Oncol. 2008;9(5):124-131.

16. Urban, N. Screening for ovarian cancer. We now need a definitive randomised trial. BMJ 1999;319(1):13178.

Source of Support: Nil; Conflict of Interest: None

Submitted: 01-12-2019; Accepted: 26-12-2019; Published online: 22-02-2020 